\title{
Effective elastic moduli of two-dimensional solids with multiple cracks ${ }^{*}$
}

\author{
ZHAN Shige（詹世革), WANG Ziqiang（WANG Tzuchiang）（王自强） \\ and HAN Xueli (韩学礼) \\ (Laboratory for Nonlinear Mechanics of Continuous Media, Institute of Mcchanics, Chinese Academy of Sciences,
} Beijing 100080, China)

Received April 22, 1998

\begin{abstract}
An accurate method which directly accounts for the interactions between different microcracks is used for analyzing the elastic problem of multiple cracks solids. The effective clastic moduli for randomly oriented cracks and parallel cracks are evaluated for the representative volume element (RVE) with microcracks in infinite medis. The numerical results are compared with those from various micromechanics models and experimental data. These results show that the present method is simple and provides a direct and efficient approach to dealing with elastic solids containing multiple cracks.
\end{abstract}

Keywords: elastic solid with multiple cracks, effective elastic modull, crack density, boundary collocation method.

Brittle materials (e. g. ceramics matrix and metal matrix composites, ceramics, rocks, and concrete) of ten contain large numbers of microcracks. Due to the presence of these microcracks, the materials become weaker and less stiff. This is of considerable interest for researchers in the fields of materials, solid mechanics, and engineering. The effective moduli of solids containing microcracks have been estimated by a number of investigators using various models, such as Taylor's models or the method of dilute distribution where microcrack interactions are entirely neglected, the self-consistent method ${ }^{[1]}$, the generalized self-consistent model ${ }^{[2]}$, and the differential scheme ${ }^{[3]}$ and the Mori-Tanaka method ${ }^{[4]}$ where microcrack interactions are indirectly accounted for. These methods are only valid for low or moderate crack density, since they do not depend on locations of microcracks, and they are not used to deal with the damage and fracture process of brittle materials. As crack density increases and microcrack spacings are closer, strong interactions between microcracks occur and the mutual positions of cracks become important. Then strong microcrack interaction models have been proposed. Kachanov ${ }^{[s]}$ proposed a pseudo traction method to solve multiple cracks problem which took into account the strong interaction between microcracks. This method is simple, and can be used to higher microcrack concentrations. But Kachanov's interaction scheme corresponds to the case in which the unknown crackline tractions are approximated only by their averages. Huang et al. ${ }^{[6]}$ calculated the effective moduli of microcracked solids, in conjunction with a unit cell model. A unit cell was assumed to be periodic in the solid. Fond et al. ${ }^{[7]}$ used the pseudo tractions technique to deal with interactions between cracks and circular cavities in two-dimensional finite or infinite media. Vavakin and

* Project supported by the National Natural Science Foundation of China (Grant No. 19704100), and the National Natural Science Foundation of Chinese Academy of Sciences (Project KJ951-1-201). 
Salganik ${ }^{[8]}$ and Carvalho and Labuz ${ }^{[9]}$ presented the results of experiments designed to measure effective elastic properties of artificially cracked and porous aluminum plates under plane stress conditions by uniaxial tension tests.

The purpose of the present study is to solve the elasticity problem of two-dimensional solid with multiple microcracks by using an accurate and efficient method ${ }^{[10]}$. Each crack is treated as continuous dislocations, and the complex potentials are expressed as series with a set of unknown coefficients. Analysis is based on a superposition scheme. By using the traction free conditions on each crack surface, a set of governing equations are formulated. The governing equations are teduced to a set of algebraic equations for the unknown coefficients and are solved numerically on the basis of a boundary collocation procedure. In the present work, crack density is a parameter that characterizes the effect of microcracking. The effective Young's moduli based on this method for randomly distributed cracks and parallel cracks are evaluated for RVE with microcracks in infinite media.

\section{Effective elastic moduli of the RVE}

\subsection{Basic formulae}

1.1.1 A single crack. It is well known that stresses and displacements for a homogeneous elastic body under plane deformation can be represented by two complex potentials. To be convenient for our purpose, potentials $\Phi(z)$ and $\Omega(z)$ will be used. Stresses can be derived from reference [11]

$$
\begin{gathered}
\sigma_{x}+\sigma_{y}=2[\Phi(z)+\overline{\Phi(z)}] \\
\sigma_{y}-i \sigma_{x y}=\Phi(z)+\Omega(\bar{z})+(z-\bar{z}) \overline{\Phi^{\prime}(z)} .
\end{gathered}
$$

A crack can be considered as a continuous distribution of infinitesimal edge dislocation. For a single crack lying on the real axis between $s=-a$ and $s=a$ in an infinite plate, the complex potentials $\Phi(z)$ and $\Omega(z)$ are given by the following formula:

$$
\Phi(z)=\Omega(z)=\frac{\mu}{\pi i(\kappa+1)} \int_{-a}^{a} \frac{g(s)}{z-s} \mathrm{~d} s,
$$

where $g(s)$ is the dislocation density at point $z=s$ on the crack surface. For the plane strain problem $\kappa=3-4 \nu$; for the plane stress problem $\kappa=(3-\nu) /(1+\nu) . \mu$ is the shear modulus and $\nu$ is Poisson's ratio.

The dislocation density can be expressed as the following series:

$$
\frac{\mu}{i(\kappa+1)} g(s)=\sum_{m=0}^{\infty} \alpha_{m} \frac{T_{m}(\xi)}{\sqrt{1-\xi^{2}}},
$$

where $T_{m}(\xi)$ is Chebyshev polynomials of the first kind and $\xi=s / a$.

Then, substituting eq. (3) into eq. (2), we obtain

$$
\Phi(z)=\Omega(z)=\sum_{m=0}^{\infty} \alpha_{m}\left(\frac{z}{a}-\sqrt{\frac{z^{2}}{a^{2}}-1}\right)^{m} / \sqrt{\frac{z^{2}}{a^{2}}-1} .
$$

Substituting eq. (4) into eq. (1), the stress field at any point due to the crack can be expressed as a series. Especially the stress field on the crack surface can be express as

$$
\sigma_{y}-i \sigma_{2 y}=\Phi^{i}(s)+\Omega^{-}(s)=-2 \sum_{m=0}^{\infty} \alpha_{m} U_{m-1}(s / a),
$$

where $U_{m}(s / a)$ is Chebyshev polynomials of the second kind. 
1.1.2 A set of arbitrary cracks. An infinite plane contains a set of arbitrary 2-D of $N$ cracks. A global Cartesian coordinate system $O x y$ is situated. A local normal-tangential coordinate system employed with origin $\left(O_{k}\right)$ at the center of the $k$ th crack is represented by $x_{k}$ and $y_{k}$. The geometry of the $k$ th crack is specified by the center coordinates $\left(x_{c}^{(k)}, y_{c}^{(k)}\right)$, orientation angle $\theta_{k}$, and the half length of the crack $a_{k}$.

Suppose that the elastic plane is subject to remote uniform loading $\sigma_{x}^{\infty}, \sigma_{y}^{\infty}$ and $\sigma_{x y}^{\infty}$. Then the traction along the $k$ th crack surface produced by it is

$$
\sigma_{y k}^{(0)}-i \sigma_{x y k}^{(0)}=2 \Gamma+\overline{\Gamma^{\prime}} \mathrm{e}^{-2 i \theta_{k}}, k=1,2, \cdots, N,
$$

where

$$
\Gamma=\left(\sigma_{x}^{\infty}+\sigma_{y}^{\infty}\right) / 4, \Gamma^{\prime}=\left(\sigma_{y}^{\infty}-\sigma_{x}^{\infty}\right) / 2+i \sigma_{x y}^{\infty} .
$$

The superposition scheme is used to solve the problem of the solids with multiple microcracks. From above, the complex potentials $\Phi_{k}\left(z_{k}\right), \Omega_{k}\left(z_{k}\right)$ and the stresses $\sigma_{x k}\left(z_{k}\right), \sigma_{y k}\left(z_{k}\right)$, $\sigma_{x y k}\left(z_{k}\right)$ produced by the $k$ th crack in the local coordinate system $O_{k} x_{k} y_{k}$ can be expanded as series.

According to the formulae of coordinate system transformation, the tractions along the $l$ th crack surface in local coordinate system $O_{l} x_{l} y_{l}$ produced by the $k$ th crack can be written as follows:

$$
\sigma_{y l}^{(k)}\left(z_{l}\right)-i \sigma_{x y l}^{(k)}\left(z_{l}\right)=\frac{1}{2}\left[\sigma_{x k}\left(z_{k}\right)+\sigma_{y k}\left(z_{k}\right)\right]\left(1-\mathrm{e}^{-2 i \theta}\right)+\left[\sigma_{y k}\left(z_{k}\right)-i \sigma_{x y k}\left(z_{k}\right)\right] \mathrm{e}^{-2 i \theta},
$$

where $\theta=\theta_{l}-\theta_{k}, \quad z_{k}=\left(C_{l}-C_{k}\right) \mathrm{e}^{-i \theta_{k}}+z_{l} \mathrm{e}^{\mathrm{i}\left(\theta_{l}-\theta_{k}\right)}, \sigma_{y^{l}}^{(k)}\left(z_{l}\right)-\mathrm{i} \sigma_{x y^{k}}^{(k)}\left(z_{l}\right)$ are the stresses produced by the $k$ th crack in the local coordinate system $O_{l} x_{l} y_{l}$.

According to the superposition scheme, the traction-free condition on each crack surface can be written as follows:

$$
\sum_{k=0}^{N}\left[\sigma_{y l}^{(k)}\left(x_{l}\right)-i \sigma_{x y l}^{(k)}\left(x_{l}\right)\right]=0,\left|x_{l}\right|<a_{l}, l=1,2, \cdots, N,
$$

where for positive $k \sigma_{y l^{\prime}}^{(k)}\left(x_{l}\right)-i \sigma_{x y^{l}}^{(k)}\left(x_{l}\right)$ are the tractions along the $l$ th microcrack surface in local coordinate system $O_{l} x_{l} y_{l}$ produced by the $k$ th microcrack. $\sigma_{y_{l}}^{(0)}\left(x_{l}\right)-i \sigma_{x y l}^{(0)}\left(x_{l}\right)$ are the tractions along the $l$ th microcrack surface in local coordinate system $O_{l} x_{l} y_{l}$ produced by the remote loading. Thus, eq. (8) is the governing equation for determining the unknown coefficients $\alpha_{k m}$ $(k=1, \cdots 2, N ; m=0,1, \cdots, \infty)$.

1.1.3 Calculation procedure. The governing equations are solved numerically on the basis of crack surface boundary collocation method. By dividing the $k$ th crack surface into $M_{k}$ elements, the collocation points on the $k$ th crack surface are given by the following expression:

$$
x_{k i}=a_{k} \cos \left(\frac{i \pi}{M_{k}+1}\right), i=1,2, \cdots, M_{k} .
$$

The infinite series can be approximated with a sufficient degree of accuracy by the corresponding truncated series with $M_{k}$ terms. The governing equation ( 8 ) is reduced to a system of linear algebraic equations for the unknown coefficients $\alpha_{k m}\left(k=1,2, \cdots, N ; m=0,1,2, \cdots, M_{k}\right)$.

Due to the closure condition at crack tips, the following equation can be given:

$$
\int_{-a_{k}}^{a_{k}} g_{k}(s) \mathrm{d} s=0
$$


It is easily shown that $\alpha_{k 0}=0$.

When the algebraic equations are solved, the complex potentials and the stress components produced by each crack are known. According to the superposition principle, the stress fields by the multiple cracks are obtained via the transformation formulae from the local coordinate systems into the global one.

\section{Effective elastic moduli of the RVE}

The effective compliance of the RVE can be calculated by the following formulae:

$$
\langle\boldsymbol{\varepsilon}\rangle=\boldsymbol{M}:\langle\boldsymbol{\sigma}\rangle \text {, }
$$

where $\langle\boldsymbol{\varepsilon}\rangle$ is the average strains, $\langle\boldsymbol{\sigma}\rangle$ is the average stress, and $\boldsymbol{M}$ is the effective compliance.

In this paper, two methods are used to calculate the average strains and stresses of the RVE.

The first method is that the average strains and stresses are calculated by means of Kachanov's method ${ }^{[5]}$. For flat cracks in $2-D$, the average strains can be expressed as

$$
\langle\boldsymbol{\varepsilon}\rangle=\boldsymbol{M}^{0}:\langle\boldsymbol{\sigma}\rangle+\frac{1}{2 A} \sum_{l}(\langle\boldsymbol{b}\rangle \boldsymbol{n}+\boldsymbol{n}\langle\boldsymbol{b}\rangle)^{l} a^{l},
$$

where $\boldsymbol{M}^{0}$ is the compliance tensor of the matrix material; $\boldsymbol{n}$ is the unit normal $\boldsymbol{n}^{(l)}$ to the $l$ th crack; $A$ is the area of the RVE, and $a^{l}$ is the half length of the $l$ th crack. $(\langle b\rangle)^{l}$ is the average displacement discontinuity vector.

The average stresses can be expressed as

$$
\langle\boldsymbol{\sigma}\rangle=\boldsymbol{\sigma}^{0},
$$

where $\boldsymbol{\sigma}^{0}$ is the remote loading.

The second method directly calculates the average strains and stresses of the RVE, which can be expressed in terms of quantities defined on the outer boundary of the RVE. The average strains are given by the integral

$$
\langle\varepsilon\rangle=\frac{1}{A} \int_{A} \varepsilon \mathrm{d} A=\frac{1}{A} \int_{C} \frac{1}{2}\left(n \boldsymbol{u}^{0}+\boldsymbol{u}^{0} n\right) \mathrm{d} l,
$$

where $\boldsymbol{u}^{0}$ is a displacement vector of the outer boundary of the RVE.

Average stresses are defined by the following formulae:

$$
\langle\boldsymbol{\sigma}\rangle=\frac{1}{A} \int_{A} \sigma \mathrm{d} A=\frac{1}{A} \int_{C} x t^{0} \mathrm{~d} l,
$$

where $\boldsymbol{x}$ is a position vector and $\boldsymbol{t}^{0}$ is a traction vector of the outer boundary of the RVE. $\boldsymbol{t}^{0}=\boldsymbol{n}$ $\cdot \sigma$, where $\sigma$ are the stresses of the outer boundary of the RVE.

For the imposed uniaxial tension in $x_{1}$-direction and $x_{2}$-direction and imposed pure shear case, respectively, we can obtain the effective compliance $M_{11}, M_{22}, M_{66}$, and then the effective moduli $E_{1}, E_{2}, \mu_{12}$ can be calculated.

\section{Numerical examples}

The crack density is defined for a microcracked solid (2-D) as ${ }^{[5]}$

$$
\rho=\frac{1}{A} \sum_{i=1}^{N} a_{i}^{2} \text {. }
$$

In this paper, $N$ is 36 , and six crack densities are assumed to be $\rho=0.10,0.15,0.20,0.25$, $0.30,0.35$. The RVE is divided uniformly into meshes in order to contain one microcrack for each mesh. Locations and orientations of microcracks are randomly generated in each mesh (for 
parallel cracks, microcracks are randomly generated in locations, but are parallel to the $x_{1}$ direction). In the course of generation, they are regenerated if there is an intersection among cracks or an intersection between microcracks and the boundary of the RVE. Fifteen sample arrays are considered for each density. The effective Young's moduli are calculated for each crack distribution for randomly oriented cracks and parallel cracks. The Poisson's ratio of the matrix material is 0.3. The present study is limited to plane stress analysis and each crack length is the same.

For randomly oriented cracks, the effective elastic moduli are evaluated and they are shown in figs. 1 and 2. For parallel cracks, the effective Young's moduli are presented in fig. 3, along with the solutions of other micromechanics models.
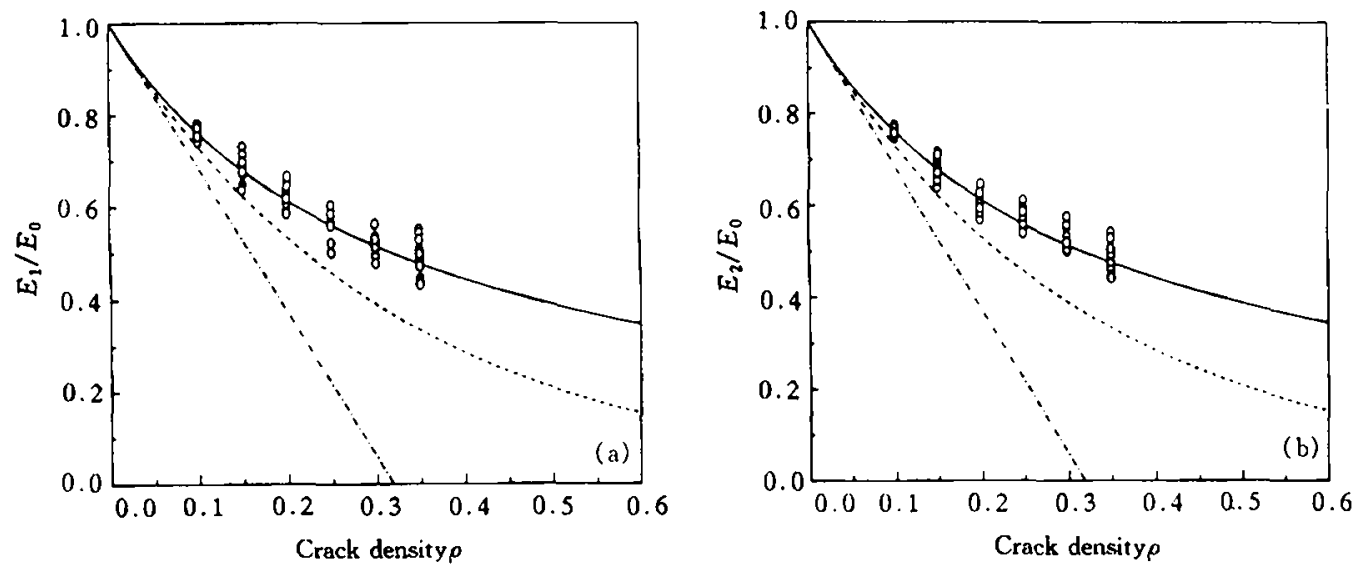

Fig. 1. Normalized effective Young's moduli vs. crack density $\rho$ for randomly oriented cracks. (a) $x_{1}$-direction; (b) $x_{2}$-direction. - Taylor's models; $-\cdots-$, differential; $-\cdots$, self-consistent; $O$, present results $(\operatorname{method} 1)$.
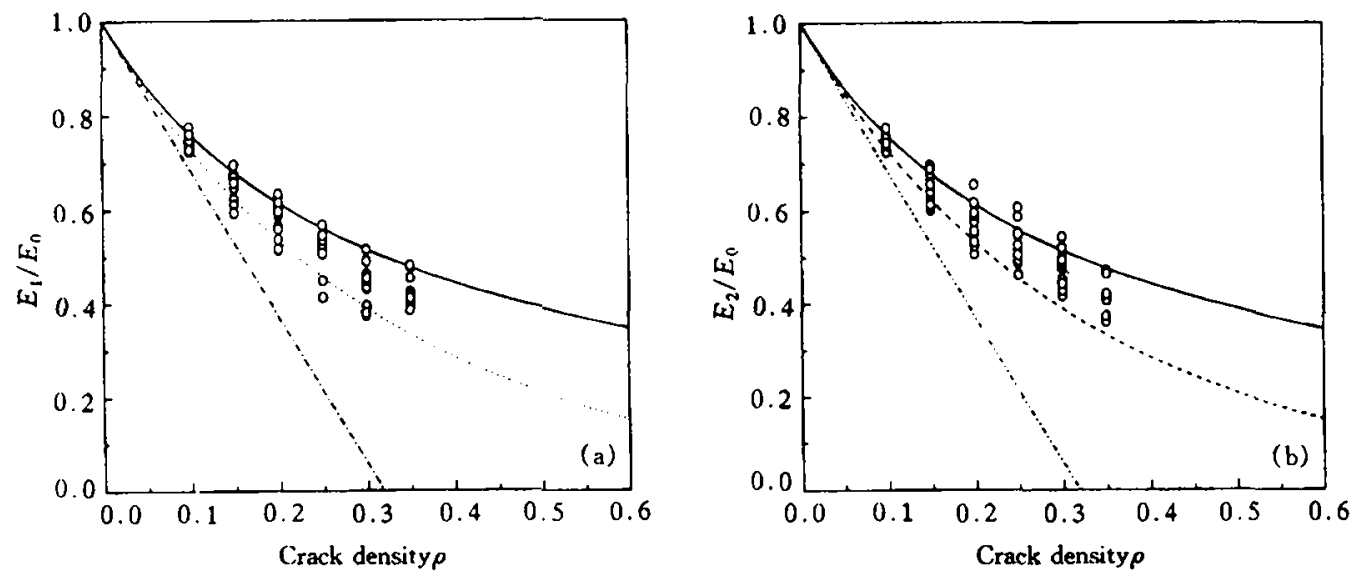

Fig. 2. Normalized effective Young's moduli vs. crack density $\rho$ for randomly oriented cracks. (a) $x_{1}$-direction; (b) $x_{2}$-direction. - . - - . - , The same as those in fig. 1; 0 , present results (method 2 ).

As shown in these figures, the 15 generalizations of microcracks for each crack density provide stochastic variations in the moduli. This scatter really reflects the features of randomly dis- 

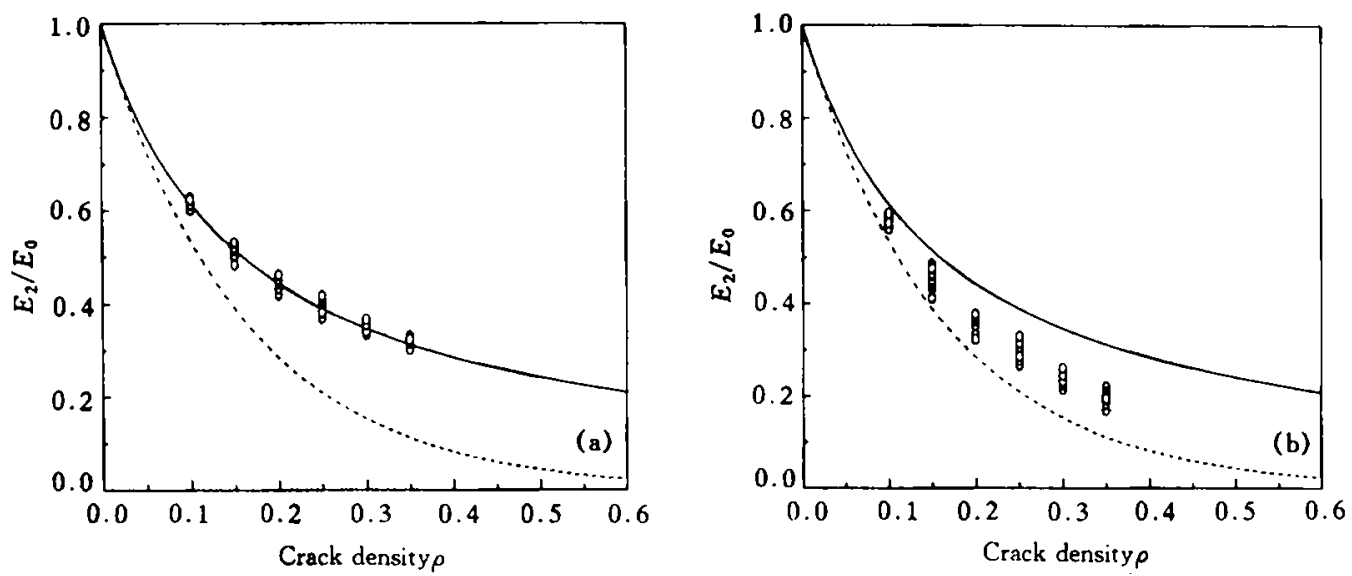

Fig. 3. Normalized effective Young's moduli vs. crack density $\rho$, for parallel cracks. - - - - - The same as those in fig. 1; 0 , present results (method 1 for (a), method 2 for (b)).

tributed cracks. For randomly oriented cracks, the mean of the moduli obtained by the first method is close to the solution using the non-interacting solution, which agrees well with that in literature ${ }^{[5]}$. The range of the moduli obtained by the second method is below that for non-interaction solution and above that for the differential method, coinciding with Huang's results ${ }^{[6]}$. For parallel cracks, the same conclusion is obtained with the randomly oriented cracks.

In order to compare the solutions of the present method with the experimental results ${ }^{[9]}$, the effective elastic moduli are also calculated for the RVE with randomly oriented cracks. The size of the RVE and the elastic constant of the material are the same as the specimens ${ }^{[9]}$. The number of the microcracks is 20 ; the crack densities are assumed to be between 0.02 and 0.15 . Fig. 4 shows the results of the present method. From the figure, it is found that the effective elastic moduli, obtained by using the second method, are closer to the experimental results ${ }^{[9]}$ than those
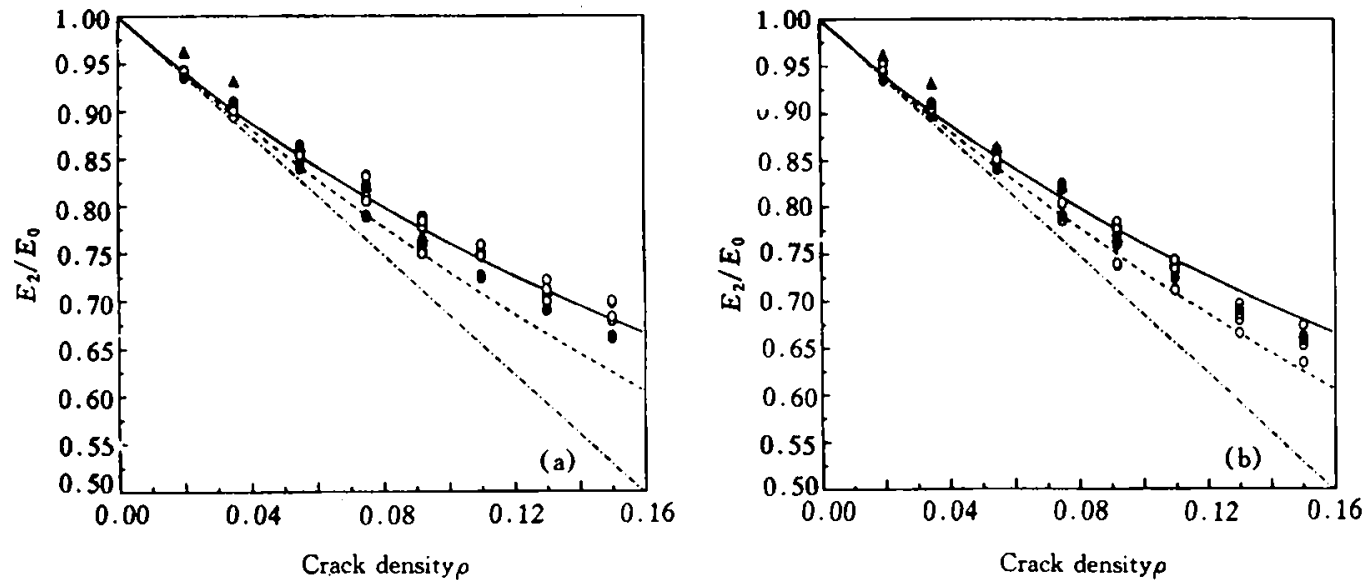

Fig. 4. Comparison of the present results with the experimental results for randomly oriented cracks. - - - - -- . The same as those in fig. 1 ; $\bullet$, experimental results $(20$ cracks $) ; \wedge$, experimental results $(6$ cracks $) ; 0$, present results (method 1 for (a), method 2 for (b)). 
of the first method, indicating that the method which directly calculates the average strains and stresses is better for representing the interactions between different microcracks and is nearer to the actual situation.

\section{Conclusion}

In this paper, for randomly oriented cracks and parallel cracks, the effective elastic moduli of the RVE are computed using the method that directly accounts for the interaction of microcracks. Numerical results show that the present method is rather general and simple. The calculated results agree well with the experimental data ${ }^{[9]}$ and other numerical results ${ }^{[5,6]}$. From the comparison of the results, it can be found that the present method has a very high efficiency and accuracy, providing an efficient approach to dealing with elastic solids containing multiple cracks.

Though this paper limits on nonintersected cracks problem, through some correction this method can cope with intersectional cracks problem. In addition, the present method can be used to solve the plane elasticity problem of finite plates with microcracks by improving it. Thus, the present method can be used not only to estimate the effective elastic properties of cracked solids, but also to study the damage procedure of brittle materials.

\section{References}

1 Budiansky, B., O'Connell, R. J., Elastic moduli of a cracked solid, Int. J. Solids Struct., 1976, $12: 81$.

2 Christensen, R. M., Lo, K. H., Solutions for effective shear properties in three phase sphere and cylinder models, J. Mech. Phys. Solids, 1979, 27: 315 .

3 Hashin, Z. The differential scheme and its application to cracked materials, J. Mech. Phys. Solids, 1988, $36: 719$.

4 Mori, T., Tanaks, K., Average stress in matrix and average elastic energy of materials with misfitting inclusions, Acta Metal., 1973, 21: 571.

5 Kachanov, M. . Effective elastic properties of cracked solids: critical review of some basic concepts, Appl. Mech. Rev., 1992, 45: 304 .

6 Huang, Y., Chandra, A., Jiang, Z. Q. et al., The numerical calculation of two-dimensional effective moduli for microcracked solids, Int. J. Solids Struct., 1996, 33; 1575

7 Fond, C., Berthaud, Y., Extensions of the pseudo tractions technique for friction in cracks, circular cavities and external boundaries; effect of the interactions on the homogenised stiffness, Int. J. Frac., 1995, 74: 1 .

8 Vavakin, A. S., Salganik, R. L., Effective characteristics of nonhomogeneous media with isolated nonhomogeneities, Izv. Akad. Nauk. SSSR, MTT, 1975, 3:65.

9 Carvalho, C. S. F., Labuz, F. J., Experiments on effective elastic modulus of two-dimensional solids with cracks and holes, Int. J. Solids Struct.. 1996, 33: 4119.

10 Han Xueli, Wang Tzuchiang, Progress in Aduanced Materials and Mechanics (eds. Wang Tzuchiang. Tsu-Wei Chou), Beijing: Peking University Press, 1996, 688-693.

11 Muskhelishvili, N. I., Some Basic Problems of Mathematical Theory of Elasticity, Groningen: P. Noordhoff, 1953. 